\title{
THE MONITORING POPULATION DENSITY OF PESTS BASED ON EDGE-ENHANCING DIFFUSION FILTERING AND IMAGE PROCESSING
}

\author{
Yuehuan Wang, Guirong Weng * \\ School of Mechanical and Electrical Engineering, Soochow University, 215021, Suzhou, \\ Jiangsu, China \\ * Corresponding author, Address: School of Mechanical and Electrical Engineering, \\ Soochow University, 215021, Suzhou, P. R. China, Tel: +86-512-67165761, Fax: +86-512- \\ 67165607,Email:wgr@suda.edu.cn
}

Abstract: As is known, agriculture is very important in China, but the problem about pests has hampered the further development of Chinese agriculture. Digital image-processing technology and mathematical morphology are referred to as the main research methods, and tiny pets like aphids among field are referred to as the research objects. Image processing technology such as edgeenhancing diffusion filtering, mathematical morphology and watershed segmentation algorithm is used to monitor pest population density, which greatly raises efficiency of pest data acquisition. After the segmentation of the image of the pests, the number of the insect individuals can be obtained from the background by using image processing technology. Computer image processing technology provides a possibility to solve this problem and becomes a very important direction to monitor regional pest population density.

Keywords: Digital Image-processing; Monitoring Population; Mathematical Morphology; Watershed Algorithm; Edge-enhancing filtering

Wang, Y. and Weng, G., 2008, in IFIP International Federation for Information Processing, Volume 259; Computer and Computing Technologies in Agriculture, Vol. 2; Daoliang Li; (Boston: Springer), pp. 899-907. 


\section{INTRODUCTION}

As is known, agriculture is very important in China, but the problem about pest has hampered the further development of Chinese agriculture. It has been long since our country has the ability to forecast plant diseases and insect pests. Nowadays, many foreign countries apply some modern information technology into application of forecasting plant diseases and insect pests (Jason W. Chapman, 2002; Minghua Zhang, 2003). However our country still applies the outdated technology. Mathematical morphology has already been widely used in all fields of image processing with the development of information technology in recent years. With the development of computer network technology, artificial intelligence, images recognition and decision support system, precision farming technology system is used more and more widely (Zhaozhi Lu, 2005; Xiaochao Zhang, 2003). Currently precision farming technology system, the research of fast data acquisition technology is far behind the researches in other fields and becomes the bottleneck of development and practice of precision farming technology system. So it is very urgent for our country to improve the level of pest data acquisition.

The analysis to plant diseases and insect pests has limitations in terms of literal descriptions, while this can be well solved by image identification. The technology of image identification is used in the fields of crop quality monitoring and crop growth state monitoring. There are less application in the field of plant diseases and insect pests monitoring. When the monitor targets are tiny pets like aphid, the survey of sampling among field will take a lot of time and pose a great threat to the veracity and reliability of the data. Using digital camera and other digital devices, we can obtain the image of pest colony, put it into computer, segment the image of the pest and get the number of the insect individuals from the background by using image processing technology. Computer image processing technology provides a possibility to solve this problem and becomes a very important direction to monitor regional pest population density (Xinwen $\mathrm{Yu}, 2001$ ). So the application of image processing technology in monitoring pest population density will improve the ability of forecasting plant diseases and insect pests and the administration skills, which is of great significance.

In this paper, image information processing technology such as edgeenhancing filtering, mathematical morphology and watershed segmentation algorithm is used to monitor pest population density, which raises efficiency of pest data acquisition greatly. 


\section{NONLINEAR ISOTROPIC DIFFUSION FILTERS}

In the last decade, PDE based models have become very popular in the fields of image processing and computer vision. The basic idea of this theory is to build nonlinear partial differential equations and use the original image as initial condition, and then the solutions in different time are the result of the filtering. Methods of this type have been proposed for the first time by Perona and Malik (Perona, Malik J, 1990).

The anisotropic form of the diffusion equation can be written as:

$$
\begin{aligned}
& \partial_{t} u(x, y, t)=\operatorname{div}(D \cdot|\nabla u|)=\operatorname{div}(g(|\nabla u|) \cdot \nabla u), t>0 \\
& u(x, y, t)=I(x, y), t=0
\end{aligned}
$$

Where $u$ is the evolving image, div is the divergence operator, $D$ is the diffusion tensor, $\nabla u=\left[u_{x} u_{y}\right]$ is image gradient norm and $g(\nabla u \mid)$ is the function of the image gradient norm, it's used to protect the image edge by adjusting the value of $|\nabla u|$, because the image gradient reflects the character of this image in some degree, the normal arithmetic edge detection is also according to image gradient. Perona and Malik method use this arithmetic, so it can remove the noise and protect the image edge information at the same time.

However, Perona and Malik method also has its problems. In theory, one should not expect is that a solution of this type is unique or stable with respect to the initial image. In practice, if the image with large amplitude noise which generates gradient value too, we will not differentiate noise from the image edge information. So the result of filtering is bad.

If there is large amplitude noise on the rim, it will cause large vibration of the image gradient and its amplitude and direction as well. All nonlinear diffusion filters that we have investigated so far utilize a scalar-valued diffusivity $D=g\left(\left|\nabla u_{\sigma}\right|\right)$ which is adapted to the underlying image structure. Therefore, they are isotropic and the flux $j=-D \cdot \nabla u$ is always parallel to $\nabla u$, we can not smooth the noise on the image edge so we should rotate the flux. These requirements cannot be satisfied by a scalar diffusivity anymore, a diffusion tensor to anisotropic diffusion filters (J. Weickert, 1997) has to be a matrix D, Weickert proposed a nonlinear anisotropic diffusion equation:

$$
\partial_{t} u=\operatorname{div}\left(D\left(\left|\nabla u_{\sigma}\right|\right) \cdot \nabla u\right)
$$

with $\mathrm{D}$ which is constructed by eigenvector and eigenvalue is a positive definite diffusivity. we choose different $\mathrm{D}$ to construct Edge-enhancing diffusion (J. Weickert, 1999) or Coherence-enhancing diffusion. 
Coherence-enhancing diffusion constructs D as:

$$
D=\left[\begin{array}{ll}
v_{1} & v_{2}
\end{array}\right]\left[\begin{array}{cc}
\lambda_{1} & 0 \\
0 & \lambda_{2}
\end{array}\right]\left[\begin{array}{l}
v_{1}^{\mathrm{T}} \\
v_{2}^{\mathrm{T}}
\end{array}\right]
$$

eigenvector: $\lambda_{1}=g\left(\left|\nabla u_{\sigma}\right|\right), \quad \lambda_{2}=1, \quad u_{\sigma}=K_{\sigma} * u, \quad$ eigenvalue: $v_{1} \| \nabla u_{\sigma}$, $v_{2} \perp \nabla u_{\sigma}$.

Weickert model ( $m=2,3$, and 4):

$$
g\left(\left|\nabla u_{\sigma}\right|\right)=\left\{\begin{array}{r}
1 \quad \text { if }\left|\nabla u_{\sigma}\right|=0 \\
1-\exp \left(-\frac{C_{m}}{\left(\left|\nabla u_{\sigma}{ }^{2} / \lambda^{2}\right|\right)^{m}}\right) \text { if }\left|\nabla u_{\sigma}\right|>0
\end{array}\right.
$$

Gaussian core: $K_{\sigma}(x)=\frac{1}{2 \pi \sigma^{2}} \cdot \exp \left(-\frac{|x|^{2}}{2 \sigma^{2}}\right)$

The value of $C_{m}$ can be obtained from the equation: $\exp \left(-C_{m}\right)\left(1+2 C_{m} m\right)=1 \quad(\mathrm{~m}=2,3$, and 4$), \quad$ so $C_{m}=2.33666,2.9183$, and 3.31488 .

The solution of polynomial (2) is put into next iterative process:

$$
\begin{aligned}
& u^{n+1}=u^{n}+\Delta t \cdot\left(\operatorname{div}\left(D^{*} \cdot u^{n}\right)\right), \\
& D^{*}=\left[\begin{array}{ll}
v_{1}\left(u^{n}\right) & v_{2}\left(u^{n}\right)
\end{array}\right]\left[\begin{array}{cc}
\lambda_{1}\left(u^{n}\right) & 0 \\
0 & \lambda_{2}\left(u^{n}\right)
\end{array}\right]\left[\begin{array}{l}
v_{1}^{\mathrm{T}}\left(u^{n}\right) \\
v_{2}^{\mathrm{T}}\left(u^{n}\right)
\end{array}\right]
\end{aligned}
$$

Where $u^{n}$ is the result of $n$ steps, $\Delta t$ is step length, $D^{*}$ is refreshed according to $u^{n}$.

\section{TOP-HAT TRANSFORMATION}

Top-Hat transformation has the property of enhancing "Gray-scale peaks" or "Gray-scale valleys" of the image signal by applying respectively the opening or closing operator (Yi Cui, 2002). Top-Hat transform can be divided into top-hat arithmetic operators and bottom-hat arithmetic operators obtained from openings or closings. Top-hat arithmetic operators are defined by: top-hat $(\mathrm{f})=\mathrm{f}-(\mathrm{f} \circ \mathrm{B})$; Bottom-hat operators are defined by: bottomhat $(\mathrm{f})=(\mathrm{f} \bullet \mathrm{B})-\mathrm{f}$. An application of Top-Hat transform is used to prevent from the effects of environmental conditions and irrelevant structural information 
and extract isolated targets and noises which have the similar shape as structural element. So the Top-Hat transformation is also a kind of high-pass filter.

\section{WATERSHED ALGORITHM OF MORPHOLOGY}

The watershed transformation is a powerful Mathematical Morphology tool for segmentation (Rafael C. Gonzalez et al., 2005). The basic principle is to think of an image as a topographic model, and suppose that water is oozing and rising at equal speed from every regional minimum, starting from the lowest one and then from each of the others as soon as the global water level reaches its altitude. Dams are built in the places where water from different minima would merge, separating the watersheds. Watershed divide lines in a gray-level image viewed as a topographic model. The dams rising above the water surface constitute the watershed divide lines, which are composed of closed contours that involve each of the regional minima and correspond to the crest lines of the relief, achieving a good segmentation by the single line.

Since watershed algorithm is sensitive to noises, images will turn out to be successive erosions after the watershed transformation and need further combination of relevant areas. The method to control successive erosions is to bring in the concept of markets. A market is the connected component of an image. Finding the market of every target is the key to solve the problem of successive erosions based on mathematical morphology.

Erosion operation is used in this image. At each step, connected components of pixels can be reduced, separated or even disappear. The residues derived from each component constitute the last erosion of the image, which is often used as marker sets for further processing.

The relevant definitions in watershed algorithm are as follows:

Iterate the image f for $\mathrm{k}$ times, $f_{k}=f \Theta k B, B$ is the unit circle, $k B$ is the circle and radius of which is $\mathrm{k}$. Ultimate erosion subset $Y_{k}$ is the element of $f_{k}$. If $l \succ k, f_{k}$ will disappear in $f_{l} . U_{k}=\left(f_{k+1} \oplus\{B\}\right) ; f_{k}$, so $Y_{k}=f_{k}-U_{k}$. If there are many targets in the image, the ultimate erosion image is: $Y=U_{k=l, m} Y_{k} . \mathrm{m}$ is the time of erosion.

\section{WATERSHED ALGORITHM BASED ON PRIORI INFORMATION}

Direct application of the watershed segmentation algorithm generally leads to over-segmentation due to noise and other local irregularities of the gradient. Over-segmentation means a large number of segmented regions. 
There are two methods to solve this problem. The first method is to smooth the gradient image to reduce to effect of noises, which directly reduces the number of segmented regions. We can also improve the morphological smoothing. We can limit the number of allowable regions by incorporating a preprocessing stage designed to bring additional knowledge into the segmentation procedure. People often aid segmentation and high-level tasks in every-day vision by using a priori knowledge. Another practical solution to this problem is to apply the watershed transformation to the image first and then incorporating relevant regions according to certain principles.

In this paper, morphology segmentation algorithm is used to diminish the influence of over-segmentation based on the priori knowledge of the size of the pests.

According to the target, set proper area thresholding a (through the methods of experiment), filter the regional objects whose.

general pixel of the regional area are less than a, eliminate the small targets of background and noises (J. Weickert, 1997).

According to the target, bring in the circular structural element ([) which is similar to the target and modify the gradient image based on the mathematical morphology (Edward R. Dougherty, 2003). The purposes of the modification are to eliminate the irregular details and noises that might lead to over-segmentation and maintain the exact orientation of the regional contour. The sense region of this structural element is the rim and adhesion area of the target.

Some targets have serious problem of adhesion. In terms of the influence of noises and adhesion, conditional extension can prevent over-segmentation effectively. Under the circumstance of $K$, ( $K$ is referred to as a limited set), conditional extension is used based on the mathematical morphology. Structural element $\mathrm{B}$ is be used to dilate image $f: f \oplus B ; K$. So $f \oplus B ; K=f \oplus B \cap K$. The experiment turns out adhesion area will tail off and be separated into many tiny areas under the use of appropriate structural element and the right radius of the structural element.

\section{APPLICATIONS}

Fig. 1 shows the pests image of aphid scanned by CCD. This paper use coherence-enhancing theory as polynomials (3), (4), (5), (6) states where: $\sigma=5, m=2, C_{m}=2.33666, \rho=5, \Delta t=0.1, t=20$. In fig. 3 the gray-scale image fusion can be done as follows: step 1: adding the original image to the image after top-hat transformation; step 2: subtracting the image after bottom-hat transformation from the result; step 3: computing the complement of the residue $k=125$. Fig. 4 shows the global thresholding 
segmentation, bwareaopen operation and regional filling operation. The original pests' image is transformed into binary image. we choose regional block, filter the noises whose general pixels of the regional area are less than 200 in the image, eliminate tiny objects in the background by using opening operation and execute regional filling operation based on morphology. Apply the distance transform to fig. 5, then apply the opening operation with '2D' 'Octagon distance' circular structural element and choose the cross structural element at the same time. Adhesion area will decrease gradually after there times of dilation operation with the radius of 6. Fig. 5 eliminates the irregular details and noises that might lead to over-segmentation and maintains the exact orientation of the regional contour. Fig. 6 shows morphology watershed segmentation. The outcome of the segmentation shows that the purpose of the segmentation is accomplished without oversegmentation according to watershed algorithm based on Priori Information. Fig. 7 shows the result of the edge detection. Fig. 8 is the calculation about the centroids of the individual image block and location of the centroids. It also shows the number of the centroids and array of the centroids (from top to bottom, from left to right). There are 12 aphids in this example and all of them are successively segregated.

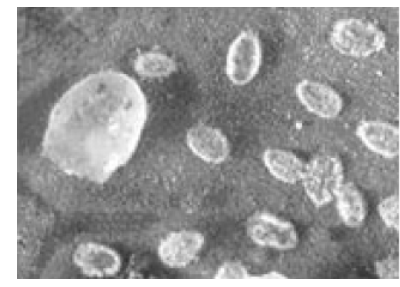

Fig. 1. Original image

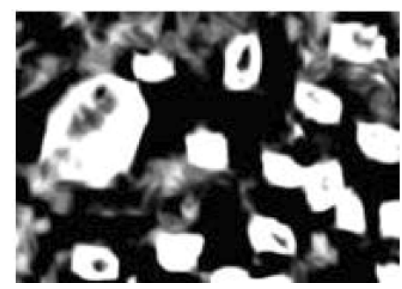

Fig. 3. Top-Hat transform

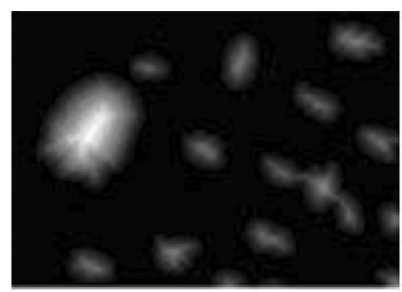

Fig. 5. Distant transform

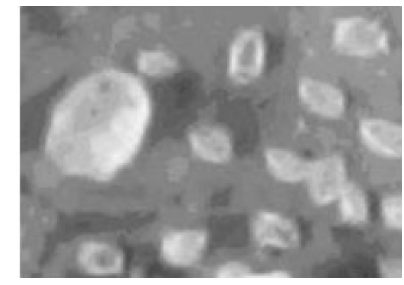

Fig. 2. Coherence enhancing filter

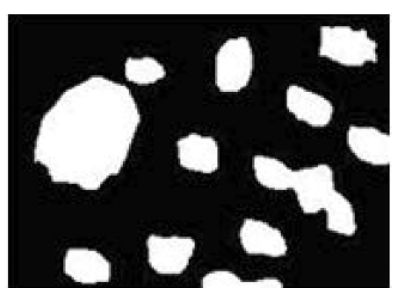

Fig. 4. Bwareaopen operation

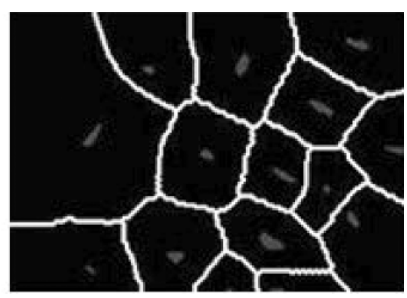

Fig. 6. Watershed segmentation 


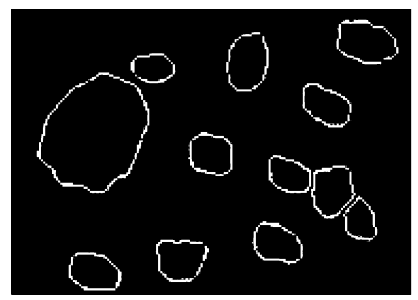

Fig. 7. Edge detection

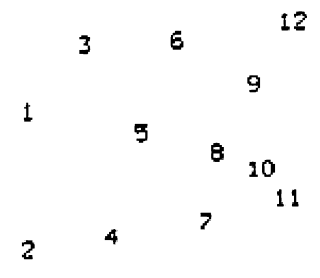

Fig. 8. The result of the statistics

\section{CONCLUSION}

After the processing given above, we can identify the exact number of the pests; restrain the effect of the similar background and noises and realize the fast collection of information from the original pests' image of aphid scanned by CCD. The efficiency of the information acquisition and the administration level will be improved a lot when edge-enhancing filtering, Top-Hat Transformation, mathematical morphology, watershed segmentation algorithm, edge detection and calculation about the centroids are used in monitoring density of the construction our country's agricultural modernization. The monitoring population density of pests based on edge-enhancing diffusion filtering and image processing has many properties such as expeditiousness and it can also save labor. This method provides convenient and convincing data for the realization of information fast collection, transmission and prevention. This research will provide some theory for the automatic administration of the crop of our country and has important practical meaning.

\section{REFERENCES}

Cui Yi. The image processing and analysis [M]. Beijing: Publishing House of Electronics Industry, 2002, 75-76, 146-149.

Edward R. Dougherty, Roberto A. Lotufo. Hands-on Morphological Image Processing [M]. SPIE PRESS July 2003, 129-136.

J. Weickert Coherence enhancing diffusion filtering [J]. International Journal of Computer Vision, 1999, 31(2/3):111-127.

J. Weickert. A review of nonlinear diffusion filtering. [J] Scale Space Theories in Computer Vision, 1997, 1252:3-28.

Jason W. Chapman, Reynolds Don R, Smith Alan D, et al. High-altitude migration of the diamondback moth Plutella xylostella to the U.K., a study using radar, aerial netting, and ground trapping [J]. Ecological Entomology, 2002, 27(26):641-650. 
Lu Zhaozhi, Shen Zuorui, Cheng Dengfa, et al. Application of information technologies in monitoring the population density of pests [J]. Transactions of the CSAE, 2005, 21(12): 112-115.

Minghua Zhang, Zhihao Qin, Xue Liu, et al. Detect ion of stress in tomatoes induced by late blight disease in California, USA, using hyperspectral remote sensing [J]. International Journal of Applied Earth Observation and Geo information, 2003, (4):295-310.

Perona, Malik J. Scale space and edge detection using anisotropic diffusion. [J] IEEE Trans Pattern Analysis Machine Intellgence. 1990, 12(7):629-639.

Rafael C. Gonzalez et al. Digital Image Processing Using MATLAB [M]. Beijing: Publishing House of Electronics Industry, 2005.9 P268-P270.

$\mathrm{Yu}$ Xinwen, Shen Zuorui. Segmentation Technology for Digital Image of Insects [J]. Transactions of the CSAE, 2001, 17(3):137-141.

Zhang Xiaochao Fang Xianfa Zhao Huaping. Information Acquisition Techniques of Precision Agriculture[J]. Transactions of The Chinese Society of Agricultural Machinery, 2002, 33(6):125-128. 